\title{
A ROBUST CHANGE DETECTOR FOR MULTILOOK POLARIMETRIC SYNTHETIC APERTURE RADAR DATA
}

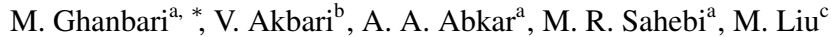

\begin{abstract}
${ }^{a}$ Department of Surveying and Geomatics Engineering, K.N.Toosi University of Technology, Tehran, Iran - mghanbari@ mail.kntu.ac.ir
${ }^{\mathrm{b}}$ Department of Physics and Technology, University of Troms $\varnothing$, Troms $\varnothing$, Norway, NO-9037 - va2akbari@ gmail.com

${ }^{\mathrm{c}}$ Key Labratory of Digital Earth Sciences, Institute of Remote Sensing and Digital Earth, Chinese Academy of Sciences, Beijing 100094, China
\end{abstract}

KEY WORDS: Change detection, SAR polarimetry, Kittler and Illingworth thresholding, Hotelling-Lawley trace statistic

\begin{abstract}
:
In this paper, we propose a robust unsupervised change detection algorithm for multilook polarimetric synthetic aperture radar (PolSAR) data. The Hotelling-Lawley trace (HLT) statistic is used as a test statistic to measure the similarity of two covariance matrices. The generalized Kittler and Illingworth (K\&I) minimum-error thresholding algorithm is then applied on the test statistic image to accurately discriminates changed and unchanged areas. The algorithm, tested on real PolSAR images, provides satisfactory results.
\end{abstract}

\section{INTRODUCTION}

Spatio-temporal data mining (STDM) is an emerging and wide research area which comprises different approaches for describing an object, an event or a position over time of interest (Julio and Adem, 2011). Detecting and mining changes occured on a spatial multitemporal database, as a part of STDM, is important in many applications such as environmental monitoring. Given a set of mutitemporal data, change mining is the problem of deriving the changing regions between any two data sets. The availability of synthetic aperture radar (SAR) data on the one hand and the insensitiveness of SAR imagery to atmospheric and sun-light conditions on the other hand offer a great potential for change detection and monitoring applications, see e.g. (Park et al., 2013, Akbari et al., 2013). In particular, polarimetric SAR (PolSAR) sensors potentially provide increased detection capability, as compared to single-polarization SAR.

In the analysis of multitemporal SAR images, several unsupervised change detection approaches have been developed and described in the literature, such as (Conradsen and Skriver, 2003, Akbari and Eltoft, 2013, Marino and Sanchez-Lopez, 2013). Unsupervised SAR change detection is generally performed in three sequential steps: 1) image preprocessing, including co-registration, geometric and radiometric corrections, and speckle reduction, 2) pixel by pixel comparison of two pre-processed SAR images by a desired test statistic, and 3) analysis of the test statistic image by applying a decision threshold resulting the final change map.

We limit our focus to change detection in multilook complex (MLC) PolSAR images, where the backscattered signal is represented by the so-called sample polarimetric covariance (or coherency) matrix. Conradsen et al., 2003, presented the Wishart likelihood ratio test (LRT) as a new test statistic for change detection in multilook PolSAR images and gave the asymptotic sampling distribution for the test statistic. Akbari et al., 2013, utilized the complex-kind Hotteling-Lawley trace (HLT) as a test statistic for change detection in multilook PolSAR images. The sampling distribution of the HLT statistic was approximated by a Fisher-Snedecor (FS) distribution. Both test statistics effectively compacts the matrix-variate information into a scalar measure.

After applying test statistic, thresholding process is performed to achieve final change/no-change map. The methods in Conradsen

\footnotetext{
${ }^{*}$ Corresponding author.
}

et al., 2003, and Akbari et al., 2013, belong to the class of constant false alarm rate (CFAR) detectors. The change detectors are performed using the approximated distribution of the test statistics at a given probability of false alarm. In the present paper, the change detector involves the optimal selection of the threshold value to accurately distinguish "change" from "no-change". The thresholding is performed using the generalized Kittler and Illingworth's minimum-error algorithm (K\&I for short) proposed in (Moser and Serpico, 2006) on the HLT test statistic image. Generalized Gamma distribution is used to model change and nochange hypotheses. This parametric model is developed because of its capability for better fitting the densities of classes in the HLT statistic image. The general block diagram of the proposed unsupervised change detection is shown in Figure 1.

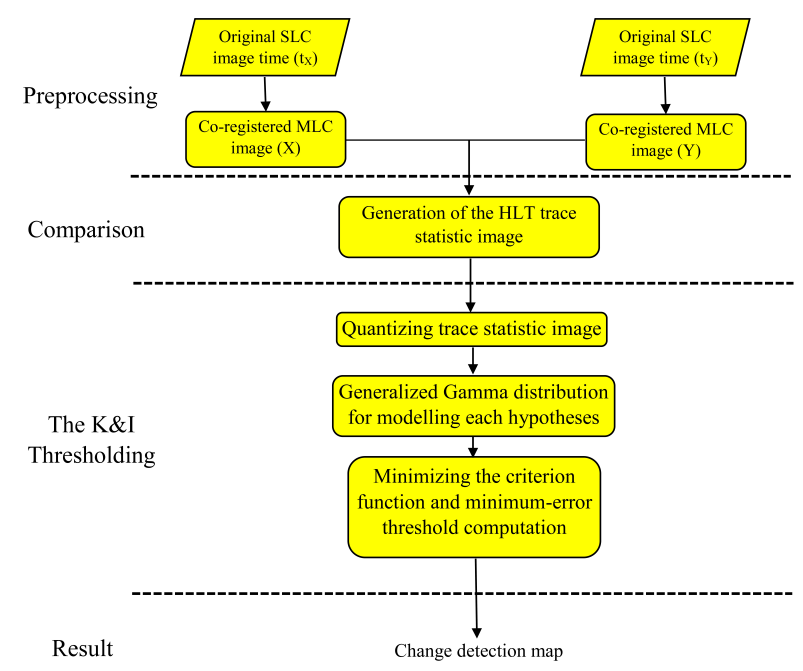

Figure 1: General block diagram of the proposed change detection approach.

The reminder of this paper is organized as follows: In Section 2., we describe the proposed change detection algorithm including the Hotelling-Lawley trace statistic and thresholding. 3. demonstrates the performance of the method with two Radarsat- 2 fully 
polarimetric images of Suzhou, China and discusses the results. Section 4 is dedicated to conclusions.

\section{THEORY}

Let $\mathcal{X}=\{\mathbf{X}(i, j) ; 1 \leq i \leq I, 1 \leq j \leq J\}$ and $y=\{\mathbf{Y}(i, j) ; 1 \leq$ $i \leq I, 1 \leq j \leq J\}$ be two co-registered multilook complex PolSAR images at times $t_{0}$ and $t_{1}\left(t_{1}>t_{0}\right)$, where $R$ and $C$ are the number of rows and columns of the images, respectively. In order to determine whether a change has occurred at position $(i, j)$ in the image, we need to compare PolSAR measurements $\mathbf{X}(i, j)$ and $\mathbf{Y}(i, j)$. These are the Hermitian and positive definite sample covariance matrices with dimension $d \times d$, where $d$ is number of polarimetric channels. We shall assume that images $X$ and $y$ have the same original resolution and have been subject to the same multilook processing resulting the same number of looks i.e., $L_{x}=L_{y}$.

\subsection{Hotelling-Lawley trace statistic}

In order to perform the change detection, one of the hypothesis should be chosen:

$$
\begin{aligned}
& H_{0}: \boldsymbol{\Sigma}_{x}=\boldsymbol{\Sigma}_{y}, \\
& H_{1}: \boldsymbol{\Sigma}_{x} \neq \boldsymbol{\Sigma}_{y},
\end{aligned}
$$

The null hypothesis $\left(H_{0}\right)$ corresponds to no-change and the alternative hypothesis $\left(H_{1}\right)$ to change. It should be noted that both hypotheses are attained by assuming $L_{x}=L_{y}$. The HLT statistic is used to measure similarity between two covariance matrices $\mathbf{X}$ and $\mathbf{Y}$ and is defined as (Akbari et al., 2013):

$$
\tau_{\mathrm{HLT}}=\operatorname{tr}\left(\mathbf{X}^{-1} \mathbf{Y}\right)
$$

where $\tau_{\text {HLT }}$ compacts the matrices $\mathbf{X}$ and $\mathbf{Y}$ into a scalar measure. HLT statistic is chosen here with respect to its clear differentiation of change and no-change cases such that it maps the no-change case to values centered around the polarimetric dimension, $d$, and the change case to values much smaller or much larger than that. It is worth noting that the HLT can be used to test either kind of change correspond to backscatter decrease and increase or even both. To achieve final change/no-change map thresholding process has been performed. In this paper we adopt Kittler and Illingworth method to perform the thresholding step.

\subsection{Thresholding}

Let $T$ be the scalar feature image whose pixels are quantized gray level values $\left\{t_{1}, t_{2}, \ldots, t_{n}\right\}$. If we suppose that these gray level values are independent identically distributed (i.i.d.) , the $\mathrm{K} \& \mathrm{I}$ addresses the problem of change detection as a two category (change and no-change) classification problem that is accomplished by minimizing a so-called criterion function. This completely automatic unsupervised approach distinguishes two hypotheses $H_{0}$ and $H_{1}$ by an optimum threshold value, $\tau$, such that the $k$ th pixel belongs to $H_{0}$ category if $t_{k} \leq \tau$ and to $H_{1}$ category if $t_{k}>\tau$.

Assuming that $h(t)$ is the histogram of the scalar feature image, K\&I method assumes the histogram as a mixture population probability density function (PDF), $p(t)$, that each components of the mixture has a given distribution with its parameters and a priori probability $P_{i}(i=1,2)$ (Kittler and Illingworth, 1986). There exists a gray level, $\tau$, for which:

$$
\begin{cases}P_{1} p(t \mid 1) \geq P_{2} p(t \mid 2), & \text { for } t \leq \tau \\ P_{1} p(t \mid 1)<P_{2} p(t \mid 2), & \text { for } t>\tau\end{cases}
$$

where $p(t \mid i),(i=1,2)$ is the no-change $(i=1)$ and change $(i=2)$ class conditional model. A conditional probability, $\varepsilon(t, \tau)$, is taken into account to replace a gray level value, $t$, by a correct scalar value given by:

$$
\varepsilon(t, \tau)= \begin{cases}-\ln P_{1 \tau}-\ln p_{\tau}(t \mid 1), & \text { for } t \in R_{1 \tau} \\ -\ln P_{1 \tau}-\ln p_{\tau}(t \mid 1), & \text { for } t \in R_{2 \tau}\end{cases}
$$

Where

$$
R_{1 \tau}=\left\{t_{1}, t_{2}, \ldots, \tau\right\}, R_{2 \tau}=\left\{\tau+1, \tau+2, \ldots, t_{n}\right\}
$$

and $P_{i \tau}$ can be estimated through histogram of the scalar feature image by this equation:

$$
\hat{P}_{i \tau}=\sum_{t \in R_{i \tau}} h(t)
$$

The function $\varepsilon(t, \tau)$, is considered as an alternative index for finding the optimal threshold. With an averaging over the whole image the criterion function can be characterized:

$$
J(\tau)=\sum_{t} h(t) \varepsilon(t, \tau)
$$

By minimizing this discriminant function the minimum-error threshold is defined, i.e. $\tau_{m}=\arg \min \left\{J(\tau): \tau=t_{1}, t_{2}, \ldots, t_{n}\right\}$.

The Generalized Gamma distribution, denoted GГD, was first proposed by Stacy (Stacy, 1962) and has been widely applied in many fields. Having three parameters this distribution has a highly flixible form and good fitting capability to the histograms of change and no-change classes. The probability density function of the GГD is given by (Krylov et al., 2011):

$$
p\left(t \mid i, \nu_{i}, \kappa_{i}, \sigma_{i}\right)=\frac{\left|\nu_{i}\right|}{\sigma_{i} \Gamma\left(\kappa_{i}\right)}\left(\frac{t}{\sigma_{i}}\right)^{\kappa_{i} \nu_{i}-1} \exp \left[-\left(\frac{t}{\sigma_{i}}\right)^{\nu_{i}}\right] \text {. }
$$

Where $\Gamma($.$) denotes the usual Gamma function and \nu_{i}$ is not zero, $\kappa_{i}$, and $\sigma_{i}$ are positive real values refer to the power, shape, and scale parameters respectively $(i=0,1)$. This probability density spans a rich and versatile family of parametric distributions in which it includes several statistical behaviors. Specifically, Exponential (for $\kappa_{i}=1, \nu_{i}=1$ ), Rayleigh $\left(\kappa_{i}=1, \nu_{i}=2\right.$ ), Nakagami $\left(\nu_{i}=2\right)$, Weibull $\left(\kappa_{i}=1\right)$, and Gamma $\left(\nu_{i}=1\right)$ are the subfamilies thus far considered in the literature. The lognormal distribution is also obtained as a limiting parametric model when $\left(\kappa_{i} \rightarrow \infty\right)$

The cruicial step of PDF parameter estimation in present paper is performed by using method of log-cumulants (MoLC). This parameter estimation method have been proposed by (Tison et al., 2004) turns out to be a well-known and precise estimation method that has been recently adopted in many literatures of the analysis and processing of SAR images (Oliver and Quegan, 2004). It is demonstrated that, applied to a selection of PDF families, MoLC enabled to obtain more feasible system of equations and applicable to a smaller sample as compared to usual method of moments, meanwhile the maximum likelihood approach is not directly applicable (Krylov et al., 2011). In the MoLC, it is proposed to exploit Mellin transform instead of usual Fourier and Laplace transforms in order to define second-kind charasteristic functions for the analysis of specifically positive-valued PDFs. Specially for a positive valued random variable, $u$, with PDF $p(u)$ defined on $u \in \boldsymbol{R}^{+}$, the Mellin transform of $p(u)$ is defined as:

$$
\Phi_{u}(s)=\int_{0}^{+\infty} u^{s-1} p(u) d u .
$$


This transform is referred to as the first second-kind charasteristic function. The second charasteristic function of the second-kind is given by the natural logarithm of $\Phi_{u}(s)$, i.e.,

$$
\psi_{u}(s)=\ln \Phi_{u}(s) .
$$

The $r$-th order second-kind moment, $\tilde{m}_{r}$, and the $r$-th order secondkind cumulant, $\tilde{k}_{r}$, is defined in the way of definition of the traditional moments and cumulants:

$$
\begin{gathered}
\tilde{m}_{r}=\left.\frac{d^{r}}{d s^{r}} \Phi_{u}(s)\right|_{s=1}=\int_{0}^{+\infty}(\ln u)^{r} p(u) d u \\
\tilde{k}_{r}=\left.\frac{d^{r}}{d s^{r}} \psi_{u}(s)\right|_{s=1} .
\end{gathered}
$$

According to (11), we call the second-kind moments as log-moment and in the same way, the second-kind cumulants are called $\log$ cumulants. For a set of i.i.d observations $\left\{x_{i}\right\}_{i=1}^{n}$ the sample estimates of cumulants can be obtained through the following relations:

$$
\begin{aligned}
& \hat{k}_{1}=\frac{1}{n} \sum_{i=1}^{n} \ln x_{i} \\
& \hat{k}_{r}=\frac{1}{n} \sum_{i=1}^{n}\left[\ln x_{i}-\hat{k}_{1}\right]^{r}
\end{aligned}
$$

where $r=2,3$. Knowing the sample cumulants, we can solve the system of equation defines MoLC by expressing sample estimates of log-cumulants as a function of PDF parameters and inverting it.

The MoLC provides for the GTD the following equations (Krylov et al., 2011):

$$
\begin{gathered}
\tilde{k}_{1 i}=\ln \sigma_{i}+\frac{\Psi\left(\kappa_{i}\right)}{\nu_{i}} \\
\tilde{k}_{2 i}=\frac{\Psi\left(1, \kappa_{i}\right)}{\nu_{i}^{2}} \\
\tilde{k}_{3 i}=\frac{\Psi\left(2, \kappa_{i}\right)}{\nu_{i}^{3}}
\end{gathered}
$$

where $\Psi(s)=(d / d s) \ln \Gamma(s)$ denotes the Digamma function and $\Psi(n, s)=\left(d^{n+1} / d s^{n+1}\right) \ln \Gamma(s)$ the $\mathrm{r}$-th order Polygamma function (Abramowitz et al., 1972). Regarding 13, the estimates $\hat{k}_{1 i}, \hat{k}_{2 i}$, and $\hat{k}_{3 i}$ is computed according to the histogram of the HLT image for each threshold value $\tau \in\left\{t_{1}, t_{2}, \ldots, t_{n}\right\}$ as follows:

$$
\hat{k}_{1 i \tau}=\frac{\sum_{t \in R_{i \tau}} h(t) \ln t}{\sum_{t \in R_{i \tau}} h(t)} \quad \hat{k}_{r i \tau}=\frac{\sum_{t \in R_{i \tau}} h(t)\left(\ln t-\hat{k}_{1 i \tau}\right)^{r}}{\sum_{t \in R_{i \tau}} h(t)}
$$

for $r=2,3$. Combining 15 and 16, we will obtain a monoton function of $\kappa_{i}$ as $\quad \tilde{k}_{2 i \tau}^{3} / \tilde{k}_{3 i \tau}^{2}=\Psi\left(1, \kappa_{i}\right)^{3} / \Psi\left(2, \kappa_{i}\right)^{2}$, which can be solved by simple numerical procedures to obtain $\hat{\kappa}_{i}$. According to 14 and 15 and the fact that $\Psi\left(2, \hat{\kappa}_{i}\right)<0$, the other two parameters are estimated by

$$
\begin{aligned}
& \hat{\nu}_{i}=\operatorname{sgn}\left(-\hat{k}_{3 i}\right) \sqrt{\Psi\left(1, \hat{\kappa}_{i}\right) / \hat{k}_{2 i}} \\
& \hat{\sigma}_{i}=\exp \left[\hat{k}_{1 i}-\left(\Psi\left(\hat{\kappa}_{i}\right)-\ln \hat{\kappa}_{i}\right) / \hat{\nu}_{i}\right]
\end{aligned}
$$

where $\operatorname{sgn}($.$) is the sign function (Abramowitz et al., 1972). It$ should be noted that the applicability condition of MoLC to GC PDF is written as (Krylov et al., 2011):

$$
\hat{k}_{2 i} \geq 0.63\left|\hat{k}_{3 i}\right|^{2 / 3} \text {. }
$$

Therefore the MoLC system of equations for the GCD case is compatible with samples that report this condition.

\section{EXPRIMENTAL RESULTS}

In order to assess the capability of the proposed approach, a real data set made up of a subset $(300 \times 233$ pixels $)$ of two Radarsat2 fully polarimetric SAR images are used. They have been acquired over the Suzhou city, China, on April 9, 2009 and June 15,2010 . The incidence angle in which the data is taken is from range $38.37^{\circ}$ to $38.95^{\circ}$. Between the two acquisition dates, we have a series of ground changes occurred in the scene due to urban expansion. The pauli decomposition images of these two PolSAR images are shown in Figure 2(a) and Figure 2(b) and the main changes are labeled by red oval in the latter Figure. A test map (see Figure 2(c)) which is made up of 3071 no-change test pixels and 1320 change pixels is available.

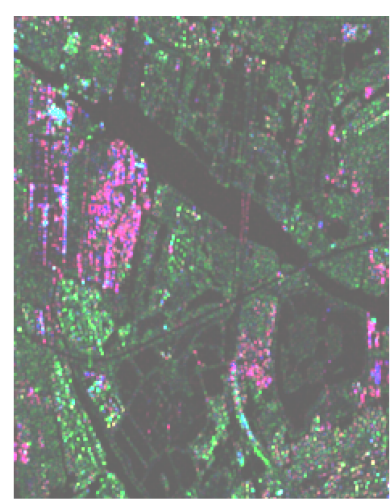

(a)

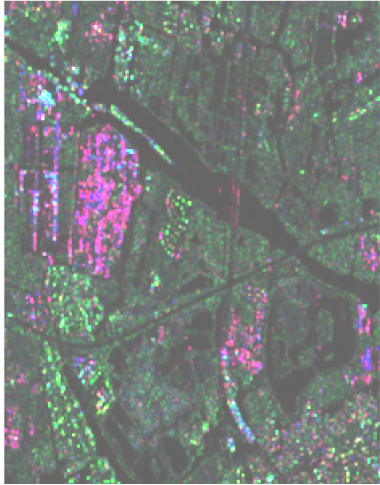

(b)

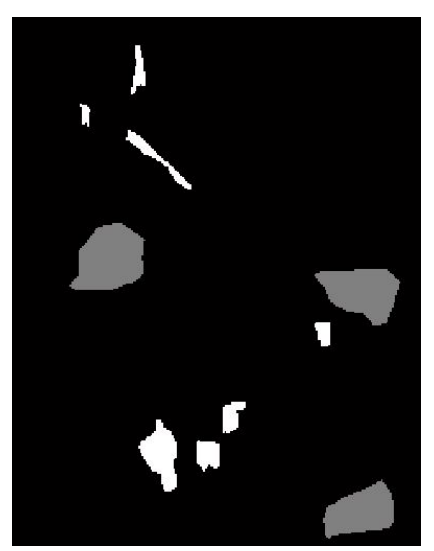

(c)

Figure 2: Multitemporal PolSAR images relating to the city of Suzhou used in this experiment. (a) April 9, 2009 pauli decomposition image. (b) June 15, 2010 Pauli decomposition image. (c) Map of changed areas (ground truth) used as reference in the experiment. Color legend for (c): white, change; gray, no-change; black, unlabeled pixels

Applying HLT statistic to this PolSAR data set, we have achieved the scalar feature images shown in Figure 3. It can be inferred from this figure that we have changes arising either radar backscatter increase (main changes occured in the scene; see Figure 3(a)) or decrease (upper right relatively white areas and the lineal structures occurred in lower left part of Figure 3(b) labeled by red oval) where adopting HLT allows for detecting both. Final change detection map was attained by implementing the proposed K\&I approach on the quantized HLT images (see Figure 4(a)) and 


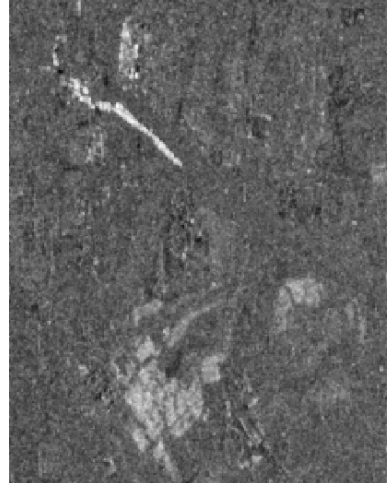

(a)

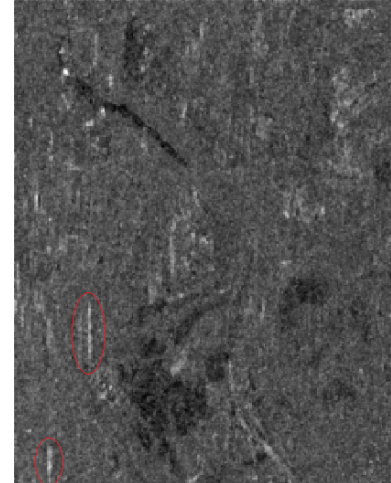

(b)
Figure 3: HLT statistic scalar feature images relating to the data set. (a) $\operatorname{tr}\left(\mathbf{X}^{-1} \mathbf{Y}\right)$. (b) $\operatorname{tr}\left(\mathbf{Y}^{-1} \mathbf{X}\right)$. Color legend: white, high values of HLT statistic followed by great changes in the direction of increase (a) and decrease (b) of the radar backscatter; gray, smaller values of HLT related to the slight changes in radar backscatter; black, least values in the HLT scalar feature image corresponding to no-change areas.

CFAR-based approach proposed in (Akbari et al., 2013) (see Figure 4(b)). Numerical results show that the proposed method gives a satisfactory detection accuracy $(87.12 \%)$ comparing to the CFAR based detection in (Akbari et al., 2013) result (85.00\%). As it can be seen in table 1 , we obtained a superior performance of the proposed generalized K\&I method based on overall error rate where the measured OER for this method is $4.17 \%$ against $4.50 \%$ in confidence level $1.00 \%$ for the CFAR-based method.

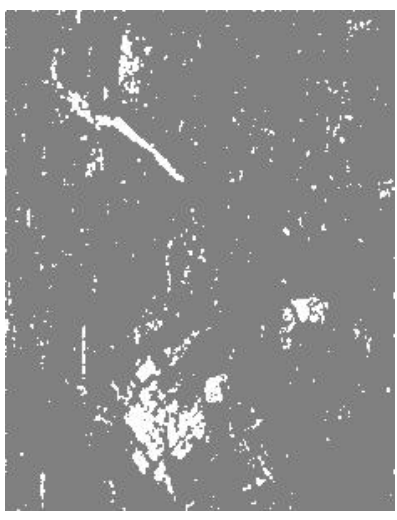

(a)

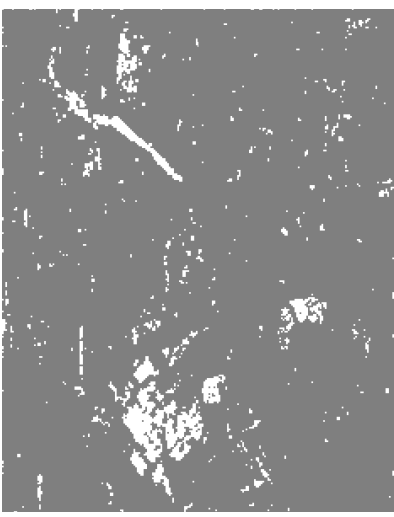

(b)
Figure 4: Final results of the unsupervised change detection for experimental data. Details of change obtained in case of (a) the generalized K\&I method proposed in this paper (b) CFAR method. Color legend: white, change; gray, no-change; black, unlabeled pixels

Table 1: Change detection performances

\begin{tabular}{|l|l|l|l|}
\hline Method & overall error rate & detection rate & false-alarm rate \\
\hline K\&I & $4.17 \%$ & $87.12 \%$ & $0.42 \%$ \\
\hline CFAR & $4.50 \%$ & $85.00 \%$ & $0.35 \%$ \\
\hline
\end{tabular}

\section{CONCLUSION}

We have proposed a novel unsupervised change detection, employing the minimum-error K\&I algorithm on the complex-kind HLT trace statistic scalar feature image. This scalar feature image was attained by applying HLT statistic to two multilook po- larimetric SAR images. Having a series of PolSAR images acquired over the same geographical area, the numerical results show that the proposed algorithm proves to be effective in mining the changes occurred between any two times of the temporal series. In particular, a comparison between the results of the proposed unsupervised algorithm and the results of the CFARbased method in (Akbari et al., 2013) confirmed the capability of the approach, as we obtained a superior performance for the proposed approach as compared to the recently proposed CFARbased algorithm. GГD PDF parameter estimation as a key stage in the process has a great effect on the final results, where if we have good-fitted PDFs to the histograms of change and no-change classes followed by an exact parameter estimation, a more accurate change detection results will be achieved; therefore we should assess and further analysis the goodness-of-fit of the estimated PDFs numerically, that would be considered for future journal work.

\section{REFERENCES}

Abramowitz, M., Stegun, I. A. et al., 1972. Handbook of mathematical functions. Vol. 1Number 5, Dover New York.

Akbari, V., Doulgeris, A. P. and Eltoft, T., 2013. Monitoring glacier changes using multitemporal multipolarization SAR images. Geoscience and Remote Sensing, IEEE Transactions on PP(99), pp. 1-13.

Akbari, Vahid, A. S. N. D. A. P. and Eltoft, T., 2013. The Hotelling-Lawley trace statistic for change detection in polarimetric SAR data under the complex Wishart distribution. Geoscience and Remote Sensing Symposium (IGARSS), 2013 IEEE International pp. 4162-4165.

Conradsen, K., N. A. A. S. J. and Skriver, H., 2003. A test statistic in the complex Wishart distribution and its application to change detection in polarimetric SAR data. Geoscience and Remote Sensing, IEEE Transactions on 41(1), pp. 4-19.

Julio, P. and Adem, K., 2011. Data mining and knowledge discovery in real life applications.

Kittler, J. and Illingworth, J., 1986. Minimum error thresholding. Pattern recognit. 19(1), pp. 41-47.

Krylov, V. A., Moser, G., Serpico, S. and Zerubia, J., 2011. On the method of logarithmic cumulants for parametric probability density function estimation.

Marino, A., C. S. and Sanchez-Lopez, J., 2013. A new polarimetric change detector in radar imagery. Geoscience and Remote Sensing, IEEE Transactions on 51(5), pp. 298-3000.

Moser, G. and Serpico, S. B., 2006. Generalized minimum-error thresholding for unsupervised change detection from SAR amplitude imagery. Geoscience and Remote Sensing, IEEE Transactions on 44(10), pp. 2972-2982.

Oliver, C. and Quegan, S., 2004. Understanding synthetic aperture radar images. SciTech Publishing.

Park, S.-E., Yamaguchi, Y. and Kim, D.-j., 2013. Polarimetric SAR remote sensing of the 2011 Tohoku earthquake using ALOS/PALSAR. Remote Sensing of Environment 132, pp. 212220.

Stacy, E. W., 1962. A generalization of the gamma distribution. The Annals of Mathematical Statistics pp. 1187-1192.

Tison, C., Nicolas, J.-M., Tupin, F. and Maître, H., 2004. A new statistical model for markovian classification of urban areas in high-resolution sar images. Geoscience and Remote Sensing, IEEE Transactions on 42(10), pp. 2046-2057. 